\title{
INVESTIGATION ON THE INTERLINK BETWEEN OPTICAL PROPERTIES OF DUSTS OVER TAIWAN AND THAR DESERT, INDIA USING CALIPSO DATA
}

\author{
M. Roja Raman ${ }^{2,1}$, Wei-Nai Chen ${ }^{2}$, Sin-Jie He ${ }^{2}$, Li-Ting Chen ${ }^{2}$, Kai-Hsuan Hsu ${ }^{2}$ \\ ${ }^{1}$ UGC-SVU Centre for MST Radar Applications, S V University, Tirupati. Andhra Pradesh, India, 517502. \\ E-mail: ramansvu@gmail.com \\ ${ }^{2}$ Research Center for Environmental Changes, Academia Sinica, Taipei, Taiwan, 115. \\ E-mail:wnchen@rcec.sinica.edu.tw
}

\begin{abstract}
The optical properties (Angstrom exponent and depolarization) of aerosols are analyzed to characterize the dust particles at different altitudes of the atmosphere using the backscatter signal from the space borne Lidar (CALIPSO) over Asian region with emphasis on Taiwan and Indian regions. The physical and optical properties of dust particles are found different at wet and dry weather conditions. The size distribution of dust particles at different altitudes is estimated. Finally the correlation between the particle depolarization and the angstrom exponent of dust particles at different altitudes is analyzed and the possible reasons are discussed. Further, the spatial inhomogeneity in the correlation coefficient is noticed and the possible relation with regional weather conditions is discussed.
\end{abstract}

\section{INTRODUCTION}

Asian dust play a major role in modulating the radiation balance by scattering and absorbing solar radiation, or modifying the cloud properties by acting as cloud condensation nuclei and ice forming nuclei in the Asiapacific region. Asian dust is known for its complex physical and optical properties with changing weather conditions. The intensive optical properties of aerosol such as Angstrom exponent and depolarization may be considered to study the dust characteristics such as size distribution and shape of the particle respectively.

Angstrom exponent $\alpha>2$ represents accumulation mode particles (Reid et al., 1964) and lower values ( $\alpha$ near 0) represent coarse mode particles such as Saharan dusts and Asian dusts (Eck et al., 1999, Sakai et al., 2002). The depolarization ratio gives the information about the sphericity of the particle. Smaller the depolarization, more spherical the particle.

The total depolarization ratio (TDP) is define as the ratio of the return light of perpendicular to parallel polariza- tions, as given by the following equation:

$$
T D P=\frac{P_{\perp}}{P_{\|}},
$$

where $P_{\perp}$ and $P_{\| \mid}$are the integrated return power for parallel and perpendicular directions relative to the outgoing laser beam. The particle depolarization $\delta_{p}$ is expressed by the following equation:

$$
\delta_{p}=\frac{R \times T D P-\delta_{m}}{R+1},
$$

where the molecular depolarization ratio $\delta_{m}$ is given as $1.4 \%$ and $R$ is backscattering ratio. The backscatter related Angstrom exponent is expressed as

$$
\alpha_{b a c k}=-\frac{d \ln \beta_{p}}{d \ln \lambda} .
$$

\section{DATA SET}

In this study, level 2 data available at $5 \mathrm{~km}$ spatial resolution from the Cloud-Aerosol Lidar and Infrared Pathfinder Satellite Observation (CALIPSO) is employed for data analysis. Depolarization ratio at $532 \mathrm{~nm}$ and backscatter based Angstrom exponent are calculatef from aerosol profiles at 532 and $1064 \mathrm{~nm}$.

1. Study Area: (refer to Figure 1.)

- Taiwan $\left(21-26^{\circ} \mathrm{N}, 119-123^{\circ} \mathrm{E}\right)$.

- Thar desert, India $\left(20-35^{\circ} \mathrm{N}, 65-80^{\circ} \mathrm{E}\right)$

2. Period of study: 2006- 2014

3. Considering only dust cases ignoring cloud and other type of aerosol particles.

4. Typical $z_{t o p}$ is $6 \mathrm{~km}$ but ranges from 4 to $8 \mathrm{~km}$, which is depend on where is the background aerosol area. Background aerosol area is choose for an area at least $500 \mathrm{~m}$ is "clean".

5. Initial backscattering ratio of background aerosol in $532 \mathrm{~nm}$ at $z_{\text {top }}$ is $R_{532}=1.05$. 
6. Initial backscatter-related Angstrom Exponent at $z_{\text {top }}$ is $\alpha_{\text {back }}=2$.

7. The selection of Lidar Ratio (LR) follows the CALIPSO inversion algorithm as suggested by Omar et al. (2009).

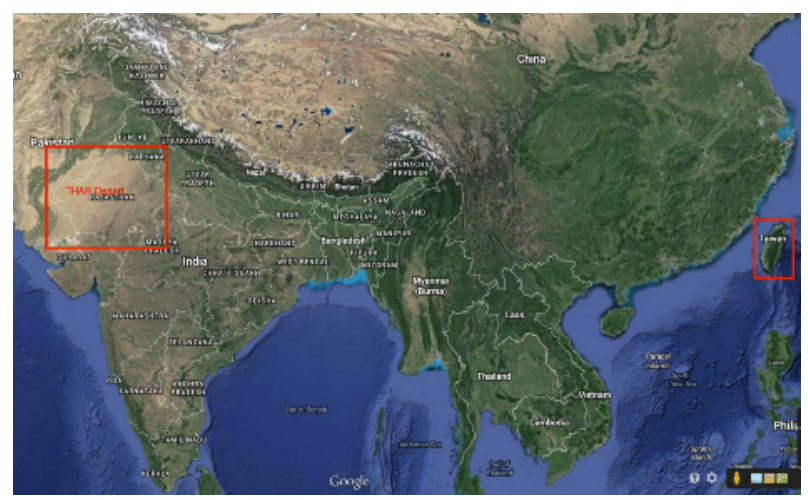

Figure 1: Geophysical location of study areas Taiwan and That desert, India.

\section{RESULTS}

A typical case of 02 March 2008 showing the higher extinction coefficient values (@532nm) up to $2 \mathrm{~km}$ at the latitudes $24-27^{\circ} \mathrm{N}$ that covers central and northern Taiwan is shown in the Figure 2. Since Taiwan region is known for occurrence of dust events during this season, the observed higher concentration of aerosol particles may be possibly Asian dust layers.

In order to confirm the possible dust layers, the Angstrom exponent is analysed. In general dust particles are in coarse mode, thus they result in lower angstrom exponent. However, the mixture of higher and lower values of Angstrom exponent at these latitudes show the particles are dominated by anthropogenic aerosols mixed with minor portion of coarse mode particles. The dust particles are generally non-spherical and show higher depolarisation. In this case the low depolarization ratio shows the domination of spherical particles with a mixture of few non-spherical particles.

Considering only dust cases ignoring other aerosol types, a negative correlation between particle depolarization and Angstrom exponent is expected, however this has not been reported experimentally so far. Here the observed negative correlation proves the theoretical hypothesis. It is also interesting to note that, the correlation turns likely positive during wet weather conditions that shows the presence of spherical like dust particles.

Further, to confirm the observed results, the possible dust source region (Thar Desert, India) has been considered where frequent elevated dust layers are observed. Similar analysis is repeated for Thar desert region and the similar negative correlation with more confidence is observed.

Occassionally, very poor correlaiton between depolarization and angstrom exponent is observed like in Figure.4, inspite of deep dust layers. This may be attributed to the signal saturation at $532 \mathrm{~nm}$.

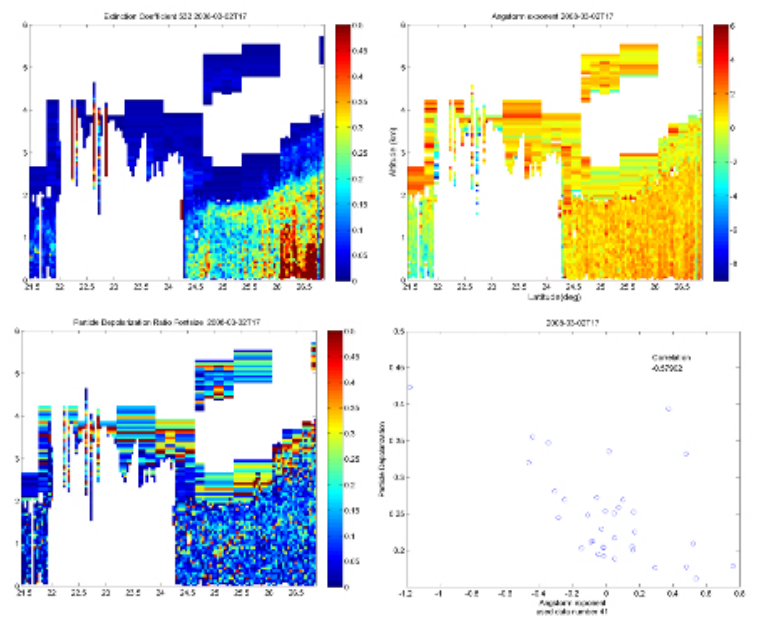

Figure 2: A typical case of CALIPSO observed Optical properties and correlation coefficient of aerosol layers over Taiwan region.
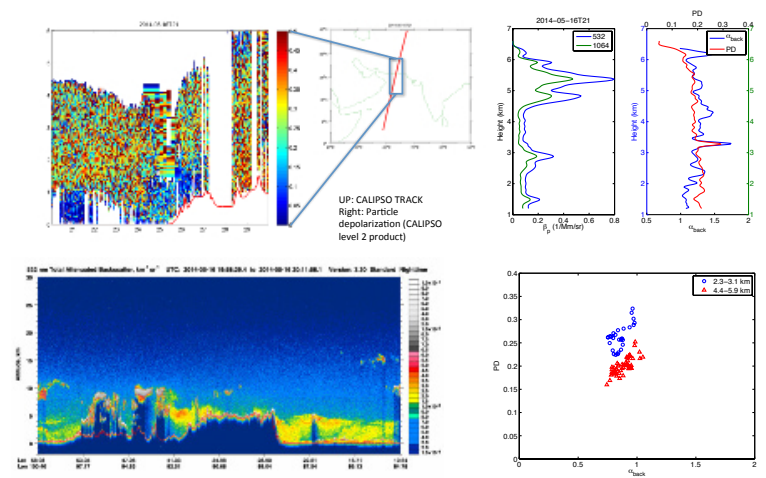

Figure 3: A dust event observed on 2014/5/16 near Thar Desert, India is select to demonstrate the calculation and interlink of dust optical properties. Attenuated backscatter is averaged from $23 \mathrm{~N}$ to $25 \mathrm{~N}$. Two distinct dust layers could be observed at 2.3$3.1 \mathrm{~km}$ and 4.4-5.9 km. It can be noticed both layer show similar $\alpha_{\text {back }} \ll 1$ but higher PD could be found in lower layer.

Considering all the dust events over Taiwan (2006-2010) and Thar desert (2014 only), the combined correlation between Particle depolarization and Angstrom exponent shows the similar negative correlation like the individual cases however with lower correlation value. 

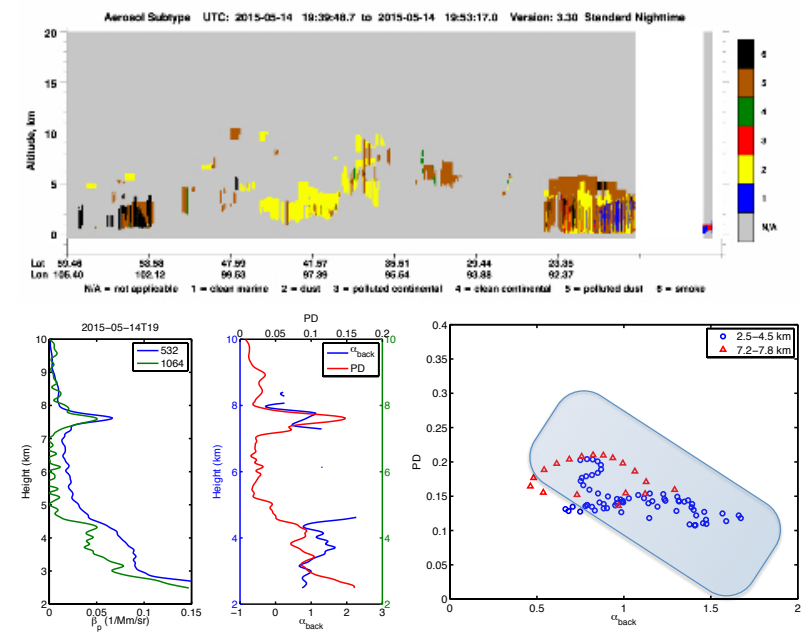

Figure 4: Similar to Fig. 3 but for Inner Mongoloa region.

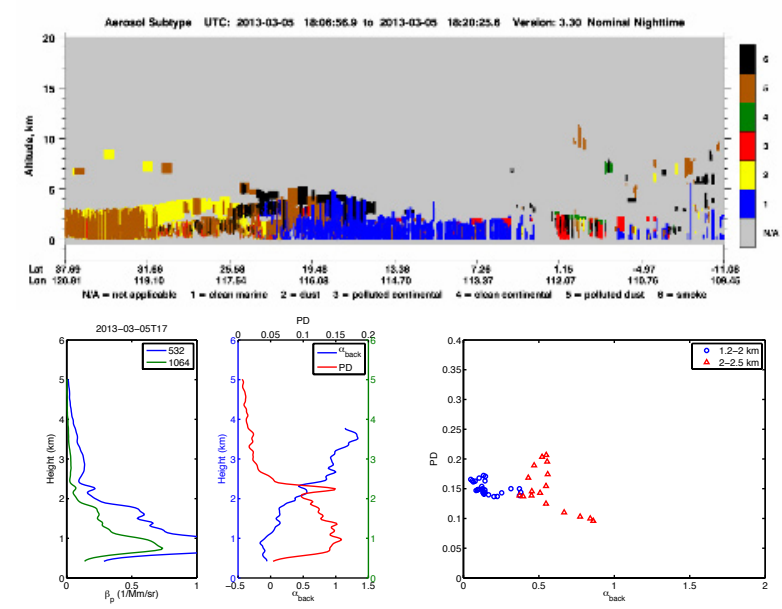

Figure 5: Similar to Fig. 3 but for IndoChina region.

This result confirms the occurrence of spherical like dust particles that may be attributed to the mixing of relatively small dust particles with the moist environment and appear like spherical particles due to moisture coating or due to external mixing with anthropogenic aerosols. More over, occassionally it is possible that the sizeparameter may be independent of depolarization ratio (Mishchenko and Sassen, 1998).

\section{SUMMARY}

- The preliminary results shows aerosols with dust layers were observed frequently over Taiwan region using CALIPSO data supporting the earlier Lidar observed dust layers over Taipei, Taiwan.

- Observed dusts have typical PD0.2 and $\alpha_{b a c k} \approx 1$.

- The correlation between PD and $\alpha_{b a c k}$ of dusts
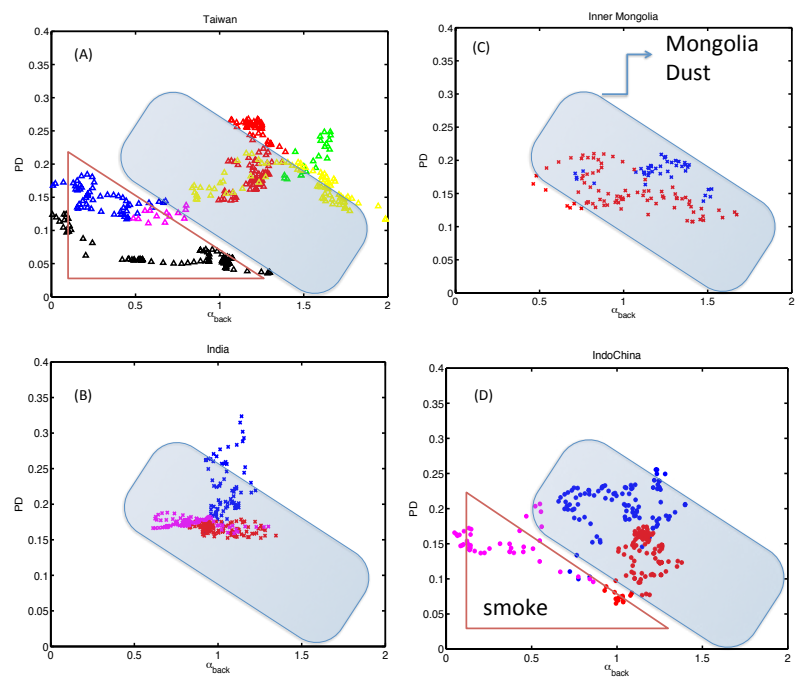

Figure 6: Results: Composed PD vs. $\alpha_{b a c k}$. (A) Taiwan: receptor of dust, smoke, and anthropogenic aerosol (B) India: dust source (C) Inner Mongolia: dust source (D) Indochina: smoke source and dust receptor.

shows a negative relation which implies that PD is dependent on particle size.

- Abnormal correlations were found especially for smokes and polluted dusts. Abnormal correlations has not been well investigated, but it might be related to mixing of dusts with water vapor according to our previous study (Chen et. al, 2007).

- The possible reason may be the condensation of water vapor on the relatively small dust particles that make the dust particle appears like spherical or small dust particles external mixing with anthropogenic aerosols.

- More detailed analysis for dusts and smokes observed by CALIPSO and ground-based lidar (include meteorological parameters) are needed to reveal the optical characteristics of aerosols.

\section{REFERENCES}

Chen, W.-N., Tsai, F.-J., Chou, C. C.-K., Chang, S.-Y., Chen, Y.-W., \& Chen, J.-P. (2007). Optical properties of Asian dusts in the free atmosphere measured by Raman lidar at Taipei, Taiwan. Atmospheric Environment, 41(36), 76987714.

Eck, T. F., Holben, B. N., Reid, J. S., Dubovik, O., Smirnov, A., ONeill, N. T., Kinne, S. (1999). Wavelength dependence of the optical depth of biomass burning, urban, and desert 
dust aerosols. Journal of Geophysical Research. doi:10.1029/1999JD900923.

Mishchenko, M. I., \& Sassen, K. (1998). Depolarization of lidar returns by small ice crystals: An application to contrails. Geophysical Research Letters. doi:10.1029/97GL03764.

Omar, A.H. et al., 2009. The CALIPSO Automated Aerosol Classification and Lidar Ratio Selection Algorithm. JOURNAL OF ATMOSPHERIC AND OCEANIC TECHNOLOGY, 26(10), pp.19942014.

Reid, J.S., Eck, T.F., Christopher, S.A., Hobbs, P.V., Holben, B., (1999). Use of the Angstrom exponent to estimate the variability of optical and physical properties of aging smoke particles in Brazil. Journal of Geophysical Research 104, 2747327490.

Sakai, T., Shibata, T., Iwasaka, Y., Nagai, T., Nakazato, M., Matsumura, T., Hamdi, S. (2002). Case study of Raman lidar measurements of Asian dust events in 2000 and 2001 at Nagoya and Tsukuba, Japan. Atmospheric Environment, 36, 54795489. doi:10.1016/S1352-2310(02)00664-7.

Zhou, J., Yu, G., Jin, C., Qi, F., Liu, D., Hu, H., Gong, Z., Shi, G., Nakajima, T., Takamura, T., (2002). Lidar observations of Asian dust over Hefei, China, in spring 2000. Journal of Geophysical Research 107, 10.1029/2001JD000802

\section{ACKNOWLEDGMENTS}

We are thankful to the National Aeronautics and Space Administration (NASA), USA for providing the the Cloud-Aerosol Lidar and Infrared Pathfinder Satellite Observations (CALIPSO) data. One of the authors Roja Raman would like to thank Academia Sinica, Taiwan for providing Post-doctoral fellowship funded by National Science Council, Taiwan. This study was supported by Taiwan National Science Council (99-2111-M-001-008MY3). 